\title{
Ground-state Properties of the One-dimensional Kondo Lattice at Partial Band-filling
}

\author{
S. Moukouri and L. G. Caron \\ Centre de recherche en physique du solide and département de physique, \\ Université de Sherbrooke, Sherbrooke, Québec, Canada J1K 2R1
}

\begin{abstract}
We compute the magnetic structure factor, the singlet correlation function and the momentum distribution of the one-dimensional Kondo lattice model at the density $\rho=0.7$. The density matrix-renormalization group method is used. We show that in the weak-coupling regime, the ground state is paramagnetic. We argue that a Luttinger liquid description of the model in this region is consistent with our calculations . In the strong-coupling regime, the ground state becomes ferromagnetic. The conduction electrons show a spinless-fermion like behavior.
\end{abstract}

PACS: 75.30.Mb, 75.30.Cr, 75.40.Mg

Typeset using REVTEX 
The heavy-fermion materials display a variety of low temperature-statest. In some compounds, the Fermi-liquid state with huge quasiparticle mass is stable down to the lowest attainable temperatures. In others, the Fermi-liquid becomes magnetic or superconductor or both. The magnetic structures vary from simple Néel antiferromagnetism through incommensurate order to ferromagnetism. This rich phenomenology arises from the interplay between a wide conduction-electron $d$-band and a partially filled narrow $f$-band of rare-earth actinide or lanthanide elements. One of the canonical models for the description of these systems is the Kondo lattice model $(K L M)$.

After almost two decades of intense studies, the $K L M$ is far from being completly understood2. Even for the simplest case of one dimension, a consensus is reached only for the half-filled case 3 . The model is an insulator. A spin gap and a charge gap open for all non-zero values of the Kondo coupling. In the metallic phase, Monte carlot and exact diagonalization 5 calculations have found that the $K L M$ presents two phases. A paramagnetic $(P M)$ state with $R K K Y$ correlations is stable at low values of the Kondo coupling. The $P M$ state evolves to a ferromagnetic $(F M)$ ground state when the coupling becomes greater than a critical value. This $F M$ phase is found to be numerically in accord with the results of strong-coupling expansion 6 . At very low densities, the model has a $F M$ phase at all non-zero couplings ture while the exact diagonalization one was restricted to a lattice of 8 sites. Therefore, the trends displayed in these computations remain to be confirmed in the ground state of much longer chains. Using standard notations the KLM may be written as follows:

$$
H=-t \sum_{i s}\left(c_{i s}^{+} c_{i+1 s}+\text { h.c. }\right)+J_{K} \sum_{i} \mathbf{S}_{i c} \cdot \mathbf{S}_{i f}
$$

where

$$
\mathbf{S}_{i c}^{\alpha}=\frac{1}{2} \sum_{s, s^{\prime}} c_{i s}^{+} \sigma_{s s^{\prime}}^{\alpha} c_{i s^{\prime}}, \mathbf{S}_{i f}^{\alpha}=\frac{1}{2} \sum_{s, s^{\prime}} f_{i s}^{+} \sigma_{s s^{\prime}}^{\alpha} f_{i s^{\prime}}
$$

with the constraint that

$$
\sum_{s} f_{i s}^{+} f_{i s}=1
$$


the letters $c$ and $f$ stand for conduction and localized electrons respectively. We applied the density-matrix renormalization group $(D M R G)$ technique to this one-dimensional Kondo lattice. We chose a density $\rho=0.7$, typical of those found in 3D compounds . We believe that the properties displayed at this density will reflect the behavior of the model in the whole region of moderate doping. Although the physical range of $J_{K}$ corresponds to small couplings, it is also interesting to study the strong-coupling regime in order to understand the behavior of the model in the whole range of parameters. We varied $J_{K}$ from 0.25 to 10 . In the $D M R G$, an iteration of the algorithm consists in adding two sites at each step. It can be immediately realized that there is a problem in keeping the electron density fixed during the iteration process. To get around such a problem, we constructed the reduced density matrix from the two states whose electron numbers bracket the desired density. In the $D M R G$ method, the states are also labelled by the $z$-component of the total spin. In the present study, we work in the subspaces having $S_{T}^{z}=0$ and $\pm \frac{1}{2}$. For more details, the reader is referred to a recent paper by Chen and one of ust, where the method has been succesfully checked for the one-dimensional $t-J$ model. The maximum lattice size we have reached is 75 with up to 180 states kept in the two external blocks. The truncation error of the Hilbert space at each iteration is around $7 \cdot 10^{-4}$ at $J_{K}=0.5$ and less than $10^{-6}$ at $J_{K}=10$. We have computed the binding energy, the magnetic structure factor, the onsite conduction electron-localized spin correlation and the electron momentum distribution.

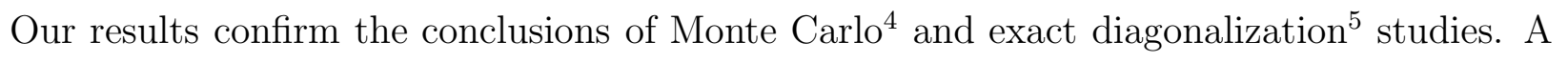
paramagnetic state is stable in the small coupling regime. This state is characterized by a maximum in magnetic structure factor at $2 k_{F_{c}}=\pi \rho$ and a singularity in the electron momentum distribution function at $k_{F_{c}}$. In the strong-coupling regime, the ground state is $F M$. The singularity of the conduction-electron momentum distribution is shifted to $2 k_{F_{c}}$.

When calculating the ground-state energy, we have taken the average of the lowest states having $\rho_{1}$ and $\rho_{2}$ such that $\rho_{1} \leq \rho \leq \rho_{2}$ (see Ref.9). This way, the ground-state energy per site of the non-interacting system can be reproduced up to four digits. In the $J_{K}=\infty$ case, the $N_{c}$ conduction electrons form perfect on-site spin-singlets with the localized spins. 
The other $N-N_{c} f$-spins remain free. The system presents a $2^{\left(N-N_{c}\right)}$ fold spin degeneracy. When the coupling is strong but finite, the ground-state energy per site is very close to $-\frac{3}{4} J_{K} \rho$. The binding energy is defined as $E_{B}=\left[E_{G}\left(J_{K}=0, N\right)-E_{G}\left(J_{K}, N\right)\right] / N$, where $E_{G}\left(J_{K}, N\right)$ is the ground-state energy. At a given density $\rho, E_{B}$ per site will be very close to $E_{\infty}=\frac{3}{4} \rho J_{K}+e_{0}$. The quantity $e_{0}=-\frac{4}{\pi} \sin \left(\frac{\pi}{2} \rho\right)$ is the energy per site of the noninteracting case. In Fig.1, we show that our results are consistent with such an analysis. The convergence to $E_{\infty}$ is very smooth. However Fig.1 also indicates that this picture breaks down around $J_{K}=2$. Below this value of the coupling, the system enters in the small coupling regime characterized by $\rho_{F} J_{K}<1, \rho_{F}$ being the density of state at the Fermi level of the non interacting Hamiltonian. For the one-impurity Kondo problem, the binding energy is given by $E_{1}=3 t\left(\rho_{F} J_{K}\right)^{2} \operatorname{Ln}(2 t)+4 t \exp -\frac{1}{\rho_{F} J_{K}} 10$. The non analytic part of $E_{1}$ defines the Kondo temperature $T_{K}$. In the inset of Fig.1, we have compared $E_{B}$ with $E_{1}$. One can see that $E_{B}$ is greater than $E_{1}$. This enhancement of the binding energy of the lattice over the one-impurity case results from the intersite magnetic interaction. However, the latter conclusion does not necessarily mean that the Kondo temperature of the lattice is greater than $T_{K}$.

The magnetic properties of the $K L M$ are studied by calculating the spin structure factors of the conduction electrons, $S_{c}(k)$ and of localized spins, $S_{f}(k)$. These quantities are defined here as follows:

$$
S_{c, f}(k)=\frac{1}{N} \sum_{l, m}\left\langle\mathbf{S}_{l, c, f} \cdot \mathbf{S}_{m, c, f}\right\rangle \exp [i(l-m) k] .
$$

At the first steps of the algorithm, $\rho_{1}$ and $\rho_{2}$ are significantly different from $\rho$ and boundary effects are non negligible. Thus, we started the calculation of the correlation functions when the lattice size was around 35 . We have noticed that the value of the correlation functions are extremely sensitive to that of $S_{T}^{z}$. We have not taken the average as for the groundstate energy because when the lowest state corresponding to $\rho_{1}$ has $S_{T}^{z}=0$, that of $\rho_{2}$ is $S_{T}^{z}= \pm \frac{1}{2}$, and vice versa. We have used only the state with $S_{T}^{z}=0$. In order to reduce the effects due to the variation of the density during the iterations, we have started the 
calculation of the correlation functions at different lattice size around 35 and then taken the average. In agreement with previous studies, we have found that the structure factor shows the competition of $P M$ and $F M$ phases. For small $J_{K}$, both $S_{f}(k)(F i g .2)$ and $S_{c}(k)$ (Fig.3) have a maximum at $2 k_{F_{c}}$. The strong spin correlation observed in $S_{f}(k)$ is due to the $R K K Y$ interaction. As the coupling is increased, this maximum flattens out. It completly disappears around $J_{K}=2$. We note that this result compares well with that of exact diagonalization and Monte Carlo studies. When $J_{K}$ is greater than this value, a new maximum arises at $k=0$. It has been shown that in the strong-coupling regime, the effective interaction between the $f$-spins is ferromagnetid 6 . The maximum at $k=0$ of $S_{c}(k)$ can be seen as a consequence of small ferromagnetic correlations tracking those of the $f$-spins.

The suppression of the $R K K Y$ correlations can be understood by the Kondo mechanism. When $J_{K}$ is small, the on-site singlet correlation $\left\langle\mathbf{S}_{i, c} \cdot \mathbf{S}_{i, f}\right\rangle$ shown in Fig.4, is different from that of perfect on-site singlets $-\frac{3}{4} \rho$. This is due to the non-local character of the Kondo singlets. This means that the singlet formed by an impurity and the conduction electrons has a spatial extension. Consequently, the RKKY interaction between impurities is favoured. As the coupling is increased, the size of the singlet reduces. It rapidly approaches that of perfect on-site singlets. As a result, the $R K K Y$ mechanism is suppressed. However contrary to the conventional view of the $K L M 12$, a global singlet state is unstable against a FM state in the strong $J_{K}$ limit. In this limit, the local Kondo singlets are mobile. It is the undelying electron motion in reduced dimension that is responsible of the $F M$ correlations.

Now, we turn to the discussion of the conduction electron momentum distribution $n(k)$. It has been argued that in the weak-coupling regime, the $K L M$ may present a $P M$ Luttinger liquid $(L L)$ structurel. The $L L$ behavior is characteristic of many one-dimensional interacting electron systems 11 . In the $L L$ theory of $P M$ systems, $S(k)$ has a maximum at $2 k_{F_{c}}$ and $n(k)$ presents a singularity at $k_{F_{c}}$. The electron momentum distribution has the form that follows:

$$
n(k)=n_{k_{F}}-C\left|k-k_{F}\right|^{\alpha} \operatorname{sgn}\left(k-k_{F}\right)
$$


We cannot calculate accurately the value of the exponent $\alpha$. But Fig.5 clearly shows that the singularity is located at $k=k_{F_{c}}$ in the weak-coupling regime. The existence of this singularity is consistent with the presence of the maximum of $S_{c, f}(k)$. So, the description of the model in the weak-coupling regime in terms of $L L$ is plausible. Further investigations of the excitation spectrum are necessary to get the full answer. At intermediate $J_{K}, n(k)$ is found to be very smooth so that it becomes hard to identify any singularity. At strong $J_{K}$ however, the singularity now appears at $k=2 k_{F_{c}}$ as in a spinless fermion system. We believe that this is due to the action of the Kondo coupling which supress a double occupancy of the conduction electrons. It freezes the electron-spin degrees of freedom. The transition from a $P M$ to a $F M$ phase does not necessary mean that the $L L$ description breaks down. It can be yet interpreted as a transition from a $P M L L$ to a ferromagnetic $L L$.

Finally, we touch upon the question of the size of the Fermi surface in the $P M$ phase. In the weak-coupling region, the $K L M$ is an effective model of the periodic Anderson model $(P A M)$. In the PAM the $f$ electrons are mixed to the conduction electron through an hybridization term. The $P A M$ is believed to have a large Fermi surface containing both conduction electrons and localized electrons. In the $K L M$, however, there is not any hybridization between the two kind of particles. It is still a matter of debate wether or not the $K L M$ has a large Fermi surface. A large Fermi surface supposes the existence of a maximum at $2 k_{F_{c}}+\pi$ in the structure factor or of a singularity in the momentum distribution function at $k_{F_{c}}+\frac{\pi}{2}$. Our results do not show any significant feature at this wave number. There is yet the possibility that the singularity at the position of the large Fermi surface is very small 13 .

In conclusion, we have used the $D M R G$ to study the $K L M$ at the density $\rho=0.7$. We believe that the behavior of the model at this density is characteristic of the moderate doping region. In agreement with previous exact diagonalization and Monte Carlo calculations, we have shown that the model presents a transition around $J_{K}=2$. The weak-coupling region is $P M$. The magnetic structure factor has a maximum at $2 k_{F_{c}}$. The electron momentum distribution function displays a singularity at $k_{F_{c}}$. We have argued that this is consistent with the description of the model in terms of $L L$. The strong-coupling region is $F M$. In 
this phase, the singularity in the electron momentum distribution is shifted to $2 k_{F_{c}}$. This can be interpreted as the presence of a ferromagnetic $L L$ structure.

We wish to thank Liang Chen for useful discussions. This work was supported by a grant from the Natural Sciences and Engineering research Council (NSERC) of Canada and the Fonds pour la formation de Chercheurs et d'Aide à la Recherche (FCAR)of the Québec government. 


\section{REFERENCES}

${ }^{1}$ G. R. Stewart, Rev. Mod. Phys. 56, 755 (1984).

${ }^{2}$ S. Doniach, Physica 91B, 231 (1977).

${ }^{3}$ H. Tsunetsugu, Y. Hatsugai, K. Ueda and M. Sigrist, Phys. Rev. B. 46, 3175 (1992); C. C. Yu and White, Phys. Rev. Lett. 71, 3866 (1993); A. M. Tsvelik, Phys. Rev. Lett. 72, 1048 (1994).

${ }^{4}$ M. Troyer and D. Würtz, Phys. Rev. B 47, 2886 (1993).

${ }^{5}$ H. Tsunetsugu,M. Sigrist and K. Ueda, Phys. Rev. B 47, 8345 (1993).

${ }^{6}$ M. Sigrist, H. Tsunetsugu, K. Ueda and T. M. Rice, Phys. Rev. B 46, 13838 (1992).

${ }^{7}$ M. Sigrist, H. Tsunetsugu and K. Ueda, Phys. Rev. Lett. 67, 2211 (1991).

${ }^{8}$ S. R. White, Phys. Rev. Lett. 69, 2863 (1992).

${ }^{9}$ Liang Chen and S. Moukouri, unpublished.

10 J. Kondo, Phys. Rev. 154, 644 (1967).

${ }^{11}$ F. D. M. Haldane, J. Phys. C 14, 2585 (1981).

${ }^{12}$ P. Fazekas and E. Müller-Hartmann, Z. Phys. B 85, 285 (1991).

${ }^{13}$ K. Ueda, T. Nishino and H. Tsunetsugu, Phys. Rev. B 50, 612 (1994). 


\section{FIGURES}

FIG. 1. The binding energy of the Kondo lattice $E_{B}$ (circles) versus the Kondo coupling. The dashed line corresponds to the strong-coupling limit. In the inset $E_{B}$ is compared with the binding energy of the one-impurity (diamonds) Kondo problem.

FIG. 2. The magnetic structure factor of the localized spins for $\rho=0.7$ at $J_{K}=0.5$ (circles), $J_{K}=1$ (diamonds), $J_{K}=2$ (stars) and $J_{K}=4$ (triangles).

FIG. 3. The magnetic structure factor of the conduction electrons for $\rho=0.7$ at $J_{K}=0.5$ (circles), $J_{K}=1$ (diamonds), $J_{K}=2$ (stars) and $J_{K}=4$ (triangles).

FIG. 4. The on-site conduction electron-localized spin correlation versus the Kondo coupling. The dashed line corresponds to the strong-coupling limit

FIG. 5. The electron momentum distribution $n(k)$ for $\rho=0.7$ at $J_{K}=0.5$ (circles), $J_{K}=1$ (diamonds), $J_{K}=2$ (stars) and $J_{K}=4$ (triangles). 
Fig. 1

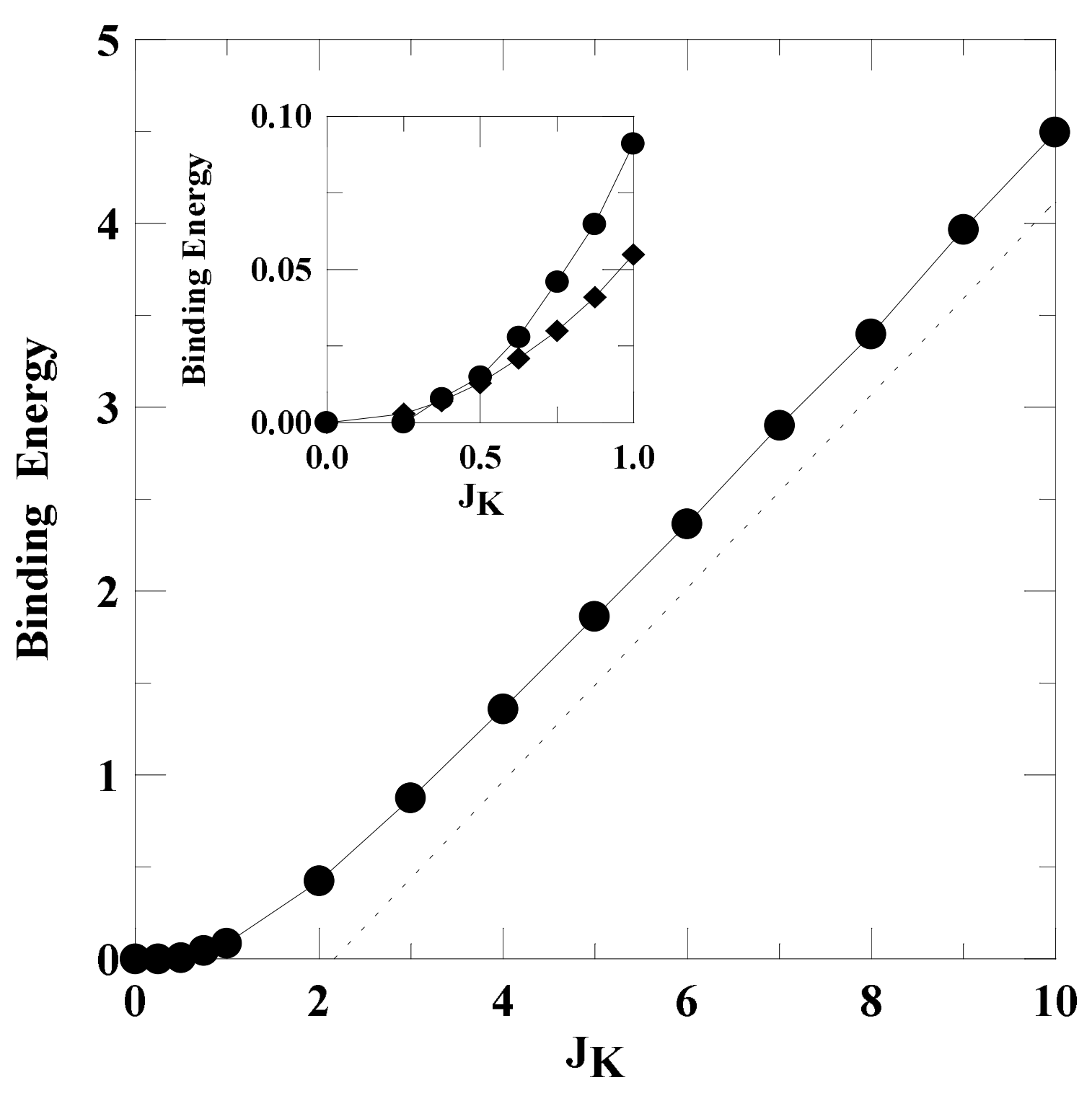


Fig. 2

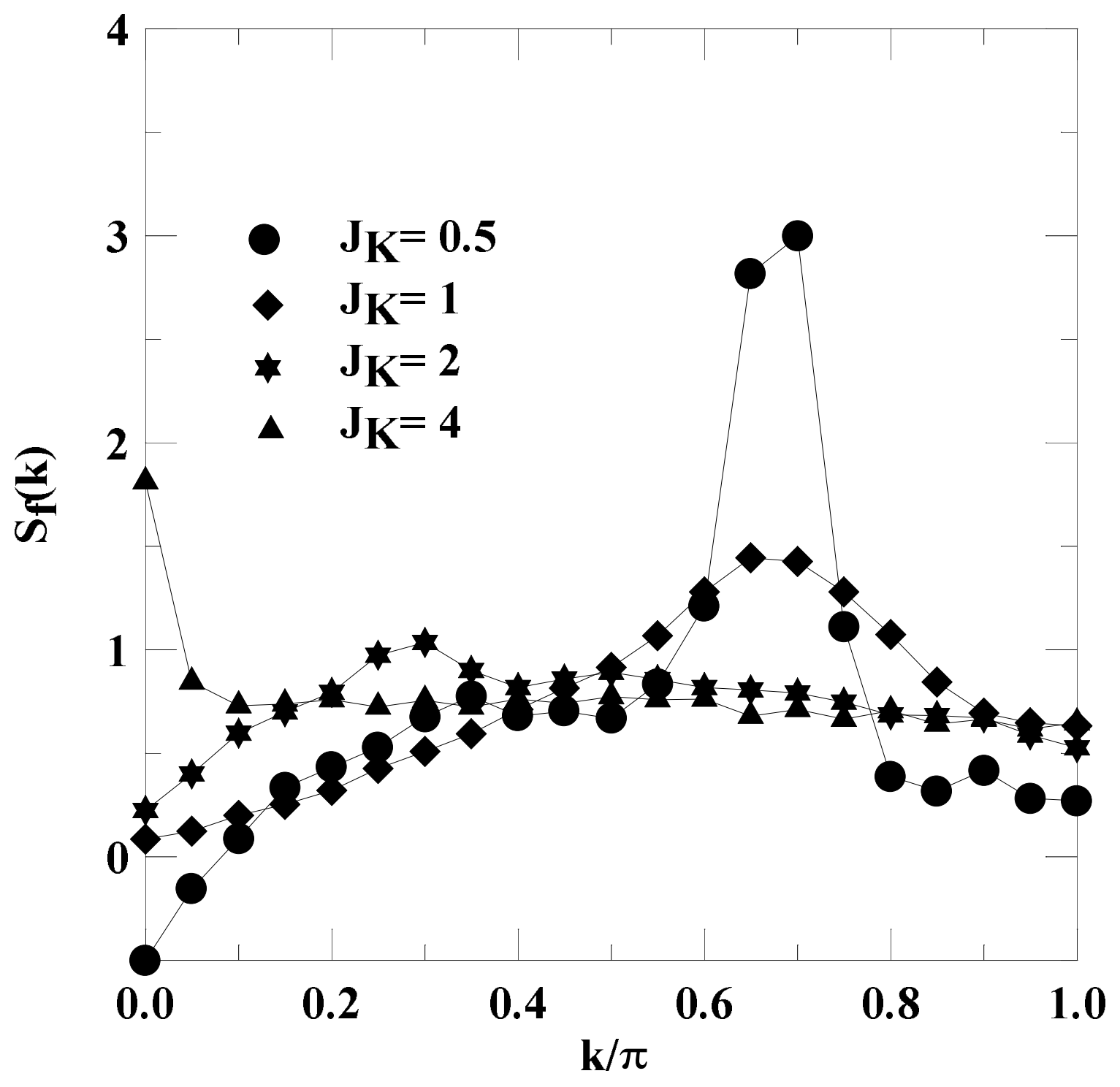


Fig. 3

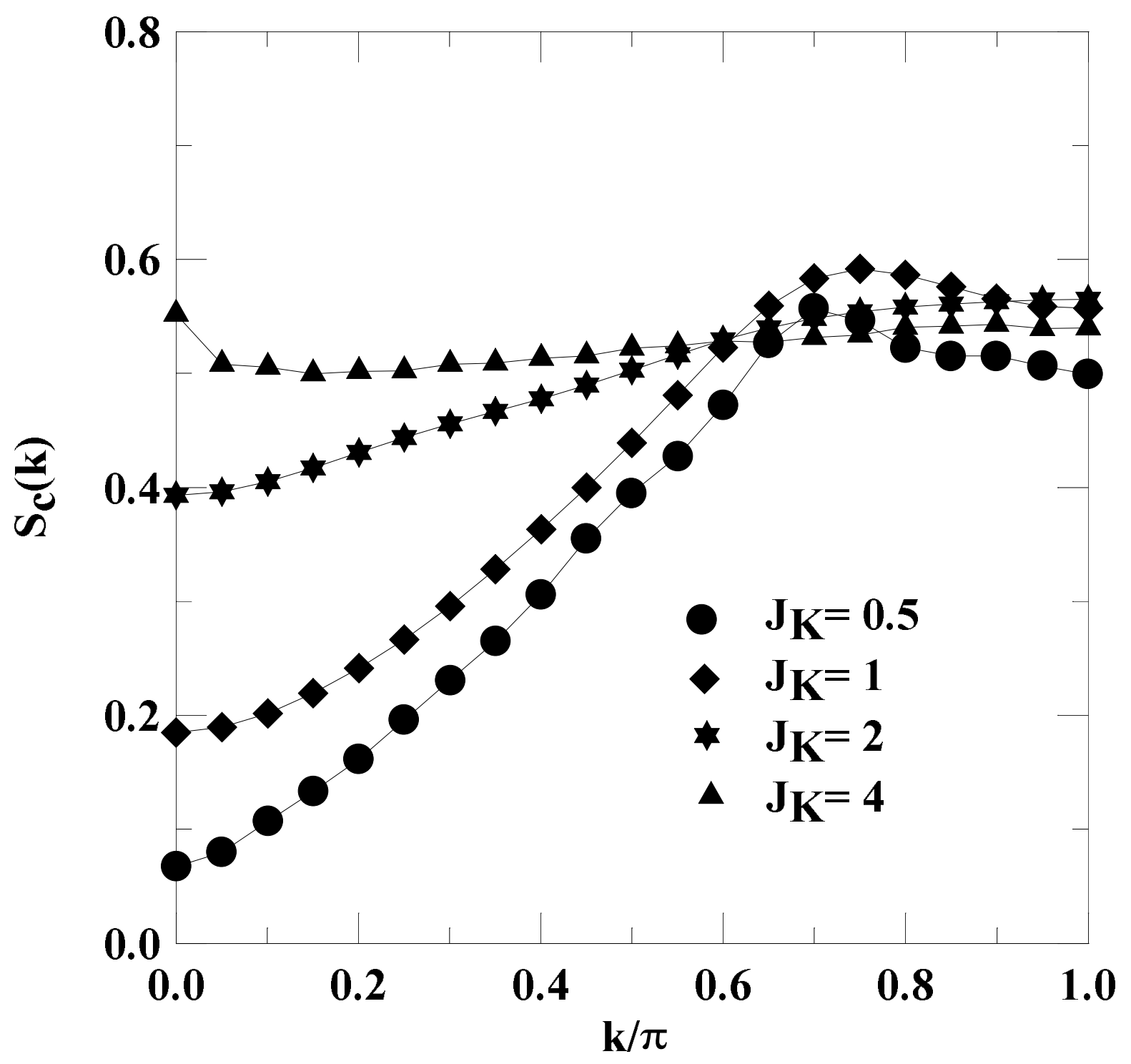


Fig. 4

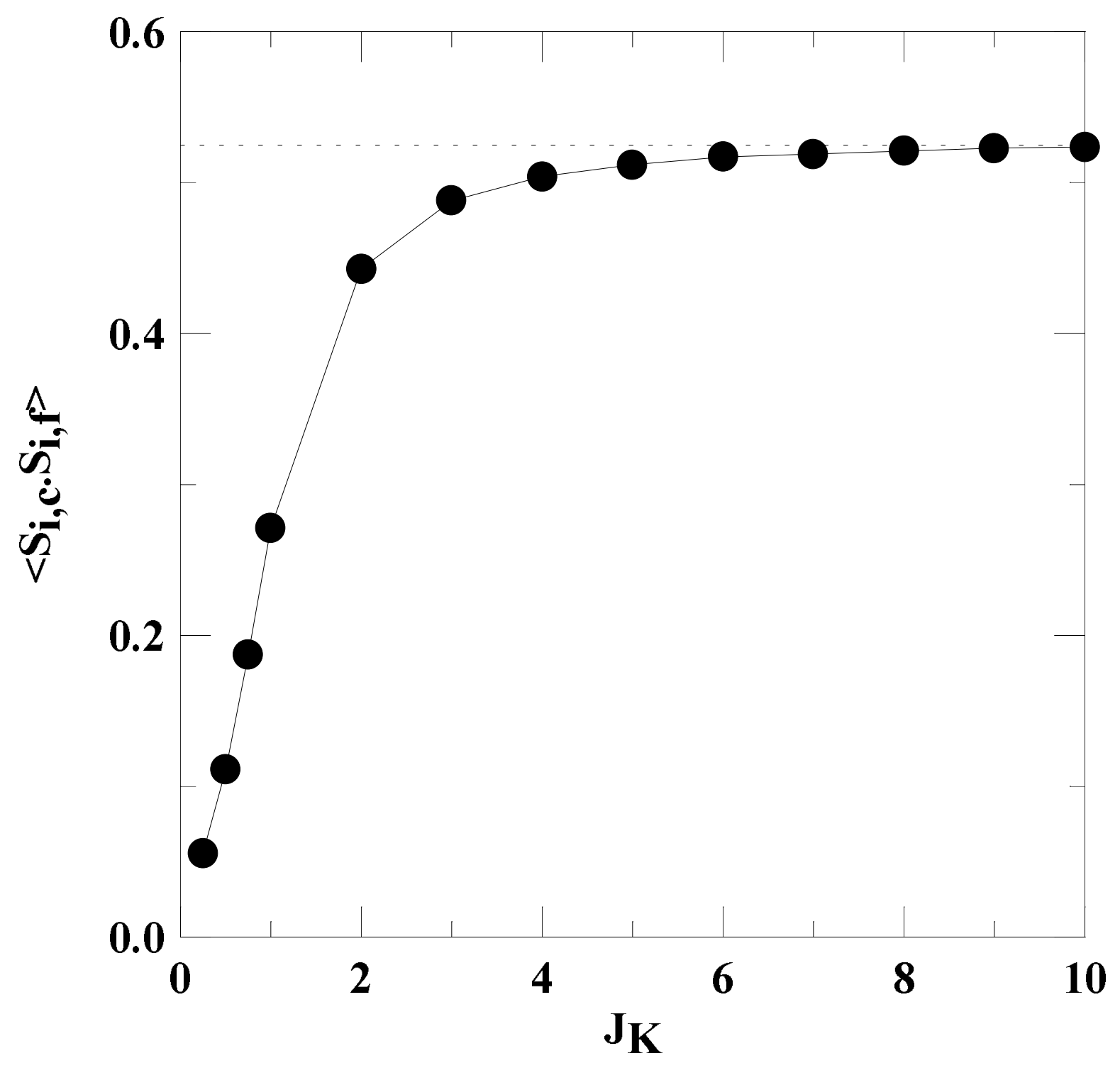


Fig. 5

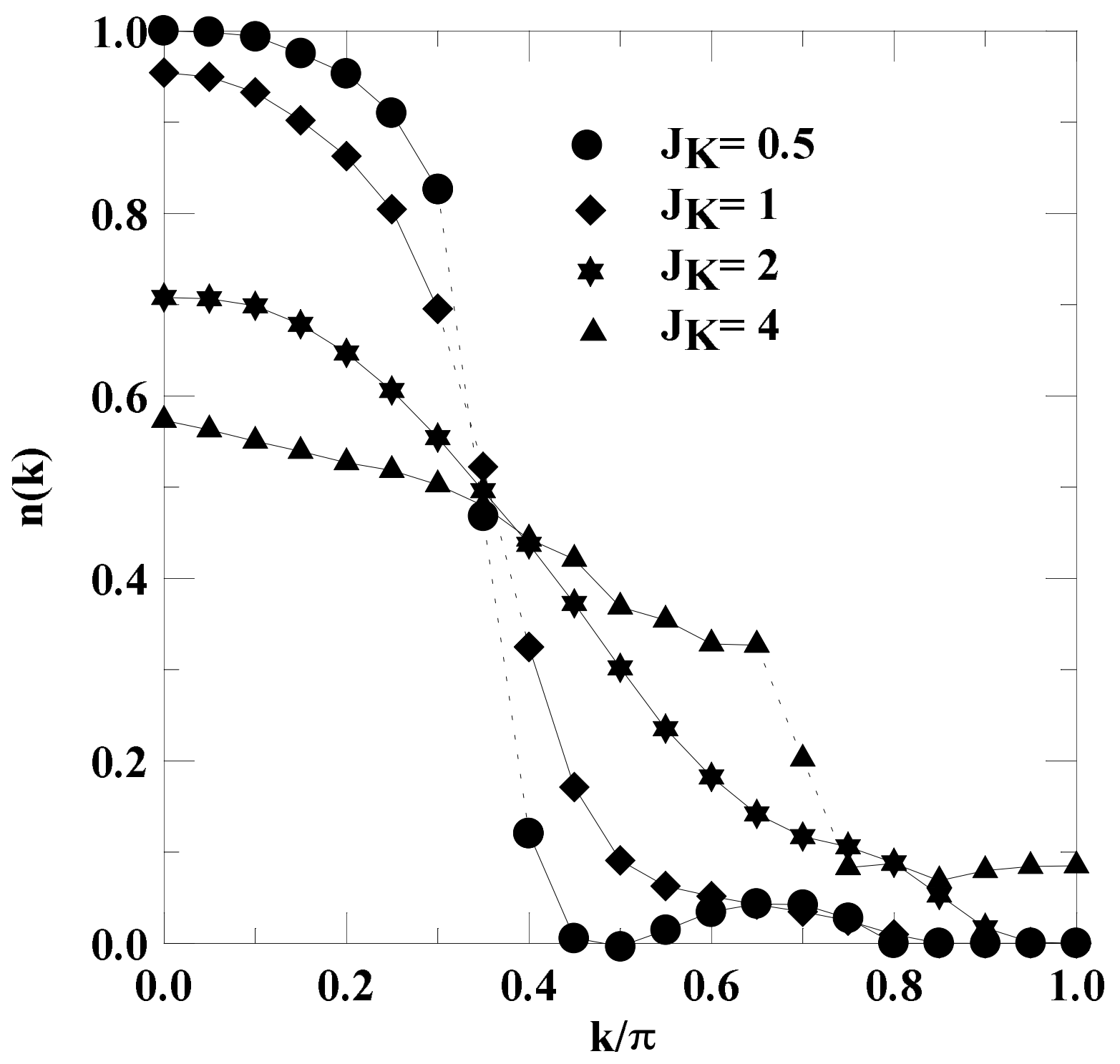

